Service social

\title{
La transformation des pratiques sociales auprès des familles en difficulté : du " paternalisme " à une approche centrée sur les forces et les compétences
}

\author{
Eve Pouliot, Daniel Turcotte et Marie-Lyne Monette
}

Volume 55, numéro 1, 2009

URI : https://id.erudit.org/iderudit/029487ar

DOI : https://doi.org/10.7202/029487ar

Aller au sommaire du numéro

Éditeur(s)

École de service social de l'Université Laval

ISSN

1708-1734 (numérique)

Découvrir la revue

Citer cet article

Pouliot, E., Turcotte, D. \& Monette, M.-L. (2009). La transformation des pratiques sociales auprès des familles en difficulté : du " paternalisme " à une approche centrée sur les forces et les compétences. Service social, 55(1), 17-30. https://doi.org/10.7202/029487ar
Résumé de l'article

Les pratiques actuelles dans le domaine des services à l'enfance sont fortement influencées par l'approche centrée sur la famille. Cette approche soutient que la famille naturelle est le milieu de vie le plus susceptible d'assurer le développement harmonieux de l'enfant, d'où la nécessité pour les services sociaux de reconnaître et de soutenir les compétences de la famille. Cette conception de l'intervention diffère de la vision jusque-là très répandue qui laisse implicitement sous-entendre que les situations d'abus et de négligence envers les enfants relèvent d'abord et avant tout des carences parentales et, donc, exigent la mise à contribution de ressources de substitution aux parents. Cette transformation de la façon de concevoir les interventions à mettre en place pour assurer la protection des enfants n'est pas strictement attribuable aux développements récents dans les référents théoriques ou idéologiques des intervenants; elle résulte également de l'évolution des mentalités en ce qui a trait au rôle de la famille et à la place de l'État dans les questions familiales. C'est ce que tente de démontrer cet article en mettant en parallèle la transformation de la famille québécoise au cours du dernier siècle, l'évolution des politiques sociales qui s'adressent à la famille et la modification des pratiques d'intervention privilégiées dans les services sociaux à l'enfance. 


\section{La transformation des pratiques sociales auprès des familles en difficulté : du " paternalisme " à une approche centrée sur les forces et les compétences}

Eve Pouliot

Ph.D. (candidate) en Service social Professeure régulière en travail social

Université du Québec à Chicoutimi Chercheure, Centre de recherche sur l'adaptation des jeunes et des familles à risque (JEFAR)

Daniel TurcotTE Ph.D., t.s., Professeure titulaire École de service social Université Laval Centre de recherche sur l'adaptation des jeunes et des familles à risque (JEFAR)

Marie-Lyne MonetTE Étudiante à la maîtrise en travail social Département des sciences humaines Université du Québec à Chicoutimi

Les pratiques actuelles dans le domaine des services à l'enfance sont fortement influencées par l'approche centrée sur la famille. Cette approche soutient que la famille naturelle est le milieu de vie le plus susceptible d'assurer le développement harmonieux de l'enfant, d'où la nécessité pour les services sociaux de reconnaître et de soutenir les compétences de la famille. Cette conception de l'intervention diffère de la vision jusque-là très répandue qui laisse implicitement sousentendre que les situations d'abus et de négligence envers les enfants relèvent d'abord et avant tout des carences parentales et, donc, exigent la mise à contribution de ressources de substitution aux parents. Cette transformation de la façon de concevoir les interventions à mettre en place pour assurer la protection des enfants n’est pas strictement attribuable aux développements récents dans les référents théoriques ou idéologiques des intervenants; elle résulte également de l'évolution des mentalités en ce qui a trait au rôle de la famille et à la place de l'État dans les questions familiales. C'est ce que tente de démontrer cet article en mettant en parallèle la transformation de la famille québécoise au cours du dernier siècle, l'évolution des politiques sociales qui s'adressent à la famille et la modification des pratiques d'intervention privilégiées dans les services sociaux à l'enfance.

Mots-clés : Transformation des pratiques sociales, intervention auprès des familles en difficulté, approche centrée sur les forces, compétences parentales.

Service social - Volume 55, numéro 1, 2009 
Current practices in the field of children's services are heavily influenced by the approach centered on the family. This approach maintains that the family is the natural living environment most likely to ensure the harmonious development of the child, hence the need for social services to recognize and support skills of the family. The design of the intervention differs from the vision who leaving implicitly understood that situations of abuse and neglect require the contribution to alternative resources for parents. This transformation of how to design interventions to be implemented to ensure the protection of children is not strictly due to recent developments in theoretical or ideological references, but also the result of changing attitudes regarding the role of the family. This article present the family in Quebec in the last century, the evolution of social policies that address family and changing practices in social services to children.

Key words: Transformation of social practices, intervention with families, strength perspective, parenting skills.

\section{INTRODUCTION}

Les pratiques actuelles dans le domaine des services à l'enfance sont fortement influencées par l'approche centrée sur la famille. Essentiellement, cette approche soutient que la famille naturelle est le milieu de vie le plus susceptible d'assurer le développement harmonieux de l'enfant, d'où la nécessité pour les services sociaux de reconnaître et de soutenir les compétences de la famille (Berg, 1996; Berg et Kelly, 2001; Rycus et Hughes, 1998). Cette conception de l'intervention diffère de la vision jusque-là très répandue qui laisse implicitement sous-entendre que les situations d'abus et de négligence envers les enfants relèvent d'abord et avant tout des carences parentales et, donc, exigent la mise à contribution de ressources de substitution aux parents. Cette façon de voir a évidemment contribué à créer une culture de l'intervention où le parent est souvent perçu comme un obstacle à la démarche d'aide, cette dernière étant davantage dirigée vers la protection et la réadaptation de l'enfant que vers le développement des forces et des compétences parentales. Par contre, dans l'approche centrée sur la famille, cette dernière est perçue comme un lieu de compétences et l'intervention, abordée comme une démarche de partenariat.

Cette transformation de la façon de concevoir les interventions à mettre en place pour assurer la protection des enfants n'est pas strictement attribuable aux développements récents dans les référents théoriques ou idéologiques des intervenants; elle résulte également de l'évolution des mentalités en ce qui a trait au rôle de la famille et à la place de l'État dans les questions familiales. C'est ce que tente de démontrer cet article en mettant en parallèle la transformation de la famille québécoise au cours du dernier siècle, l'évolution des politiques sociales qui s’adressent à la famille et la modification des pratiques d'intervention privilégiées dans les services sociaux à l'enfance. Dans un souci de synthèse, cette transformation sera 
découpée en trois grandes phases ${ }^{1}$ qui sont associées à la « société traditionnelle », la « société moderne » et la « société actuelle » ${ }^{2}$.

\section{La société traditionnelle et la naissance des pratiques sociales pour la famille}

Dans le Québec traditionnel, la famille est vue comme la pierre angulaire de la société. Bien que le clergé et les élites locales influencent les valeurs et les normes, la famille demeure une entité essentiellement privée au sein de laquelle une importance particulière est accordée à l'autorité paternelle (Laurendeau, 1985). Mais au-delà de l'autorité formelle, les mères ont une grande influence dans les prises de décision et l'organisation des relations familiales (Fortin, 1987). Elles sont les principales responsables de l'éducation des enfants et leurs pratiques éducatives se basent essentiellement sur la coutume et le "gros bon sens » (Laurendeau, 1985). Étant donné que les familles sont nombreuses ${ }^{3}$ et que les femmes ont une multitude de tâches à accomplir pour assurer le bon fonctionnement de l'entreprise familiale, l'éducation des enfants est teintée de discipline rigoureuse et de fermeté (Chabot, 1989). De plus, la période de maternage est très courte et les enfants passent rapidement de la petite enfance à l'univers des adultes, de façon à ne pas rester longtemps un fardeau économique pour leurs parents (Ariès, 1973; Provencher, 1988). Les problèmes sociaux sont perçus comme le résultat de déficits individuels et les formes de marginalité familiale concernent principalement l'abandon du foyer, les naissances illégitimes, l'abandon des enfants, la violence ainsi que l’alcoolisme.

\section{Une présence réduite des services sociaux publics}

Devant le caractère privé de la famille et le peu d’importance accordée à la période de l'enfance, la présence de l'État dans la sphère familiale est limitée et son contrôle sur les pratiques éducatives est pratiquement inexistant. En effet, au cours des trois premiers siècles de colonisation, les problèmes qui préoccupent la société sont la maladie et l'indigence, et ces problèmes relèvent d'abord et avant tout des responsabilités individuelles et familiales. Selon

1. Cette typologie présente des «types idéaux » de société, c’est-à-dire des modèles construits par les chercheurs pour rendre compte des changements sociaux, économiques, politiques et culturels qui marquent le passage de l'une à l'autre. Ces types sont "idéaux » dans la mesure où ils ne correspondent pas entièrement et parfaitement aux réalités rencontrées et observées directement dans la vie sociale. Ils sont cependant utiles afin de comprendre les changements qui ont modifié l'institution de la famille et les pratiques sociales au sein de la société québécoise à travers différentes époques. Les trois "types idéaux » présentés dans ce document couvrent donc des périodes historiques qui ne sont pas mutuellement exclusives et qui se chevauchent fréquemment. À titre de repère général, notons que les études historiques interprètent le passage du Québec traditionnel à sa modernisation comme une lente évolution qui débute au tournant du $\mathrm{XX}^{\mathrm{e}}$ siècle et qui culmine lors de la Révolution tranquille des années 1960. La « société actuelle » réfère, quant à elle, aux années 197080 jusqu'à nos jours.

2. Dans le texte, nous parlons de «société actuelle » étant donné que les théoriciens ne s'entendent pas en ce qui concerne la terminologie à utiliser pour parler de la société dans laquelle nous évoluons actuellement.

3. Ce portrait global ne traduit pas toutes les nuances qui existent entre les familles québécoises, notamment entre le profil socio-économique des familles. Il s'agit d'un portrait général qui traduit un profil type et non l'ensemble des familles. 
D’Amours (1986), la famille constitue alors la base des services d'entraide et, à défaut de ressources au sein de la cellule familiale, le voisinage et la paroisse prêtent assistance. L'État intervient peu dans les problèmes familiaux et, à moins de disettes graves ou d'épidémies, son action se déploie surtout en ce qui concerne la santé publique et la mortalité infantile ${ }^{4}$ (Mayer, 2002). D’ailleurs, ce sont les médecins qui vont s’introduire en premier dans les foyers québécois en lançant une campagne d'éducation populaire auprès des mères et en poussant le gouvernement à réglementer les conditions sanitaires (Laurendeau, 1985). Il s’agit alors essentiellement d'une action qui vise les familles qui vivent dans des conditions matérielles très difficiles. Règle générale, l'assistance à l'enfance est prise en charge par l'Église et se structure principalement autour de la paroisse, du voisinage et des associations de charité (Carisse, 1974; Mayer, 2002). L’État n’intervient qu'épisodiquement pour accorder une reconnaissance juridique aux institutions et suppléer à l'insuffisance de dons par des subventions ponctuelles (Mayer, 2002).

À compter de la deuxième moitié du XIX ${ }^{\mathrm{e}}$ siècle, la protection de l'enfance se dissocie de l'assistance charitable et différentes législations propres à l'enfance entrent en vigueur (D’Amours, 1986). Adopté par la Législature de Québec en 1869, l’Acte concernant les écoles d'industrie et de réforme constitue la première véritable intervention spécifique du législateur québécois à l'égard de l'enfance abandonnée. Selon Joyal (2000), l'État ne cherche pas alors à contrôler ou à modifier l'exercice de la puissance paternelle, mais vise plutôt la formation et le redressement de l'enfant par l'imposition d'un cadre disciplinaire rigide et l'apprentissage d'un métier. L'État intervient pour se substituer à la famille «lorsqu'elle n’existe pas, qu'elle a délaissé l'enfant, ou encore pour renforcer l'autorité du père de famille ou du directeur d'institution » (Joyal, 2000, p. 41). Par ailleurs, devant la dénonciation, émanant principalement du milieu syndical, des abus du machinisme industriel, l'État impose certaines normes relatives à l’âge, aux heures de travail et à la sécurité dans les manufactures; des dispositions sont adoptées afin de favoriser la scolarisation des enfants québécois et l'âge minimum des jeunes travaillant dans les usines est fixé à 14 ans. Donc, il ne s’agit pas tant de s’ingérer dans la famille que de réglementer la place des enfants dans la société, et plus particulièrement, au sein du marché du travail. Malgré les quelques incursions de l'État dans la vie des familles traditionnelles, l'aide aux familles demeure de prédominance philanthropique. C'est la crise des années 1930 qui va marquer l'éclatement du système de charité paroissial et le début des agences de service social.

\section{Des pratiques sociales calquées sur le modèle médical}

La Révolution industrielle a marqué l’apparition de nouveaux problèmes sociaux, tels que la pauvreté, l'insalubrité des logements et la criminalité, forçant l'État à intervenir. Selon Mayer (2002), la naissance du service social est donc attribuable aux mouvements d'industrialisation et d’urbanisation dont il essaie d'atténuer les conséquences les plus criantes; son action est donc

4. Jusqu'au tournant du $\mathrm{XX}^{\mathrm{e}}$ siècle et même par la suite, Montréal connaît un taux de mortalité infantile extrêmement élevé : plus d'un enfant sur quatre n’atteint pas l'âge d'un an (Joyal, 1999). 
essentiellement dirigée vers la classe ouvrière. La culture de l'époque joue un rôle important dans la définition des pratiques sociales. Le travail social de l'époque n'est pas à proprement parler une " profession » et encore moins une « science », mais davantage un « art » qui s'inspire des valeurs chrétiennes. Cependant, de nouveaux courants de pensée, dont ceux du scientisme et du libéralisme, transforment graduellement l'intervention sociale et amorcent la professionnalisation du service social. Une nouvelle façon de concevoir la charité est d'abord mise en pratique en Angleterre par la Charity Organization Society (COS) à la fin du XIX siècle. Quelques décennies plus tard, le mouvement apparaît aux États-Unis et au Canada. Aux actions d'éducation et de mobilisation qui prennent forme dans les Settlements s'ajoute alors une conception du travail social axée davantage sur l'aide individuelle. Diffusé initialement par Mary Richmond dans Social Diagnosis (1917), le travail social individuel calque son action sur celle de la profession médicale et tend à traiter les problèmes sociaux à l'instar d'une maladie. Essentiellement centrée sur les déficits des individus et des familles à aider, cette orientation basée sur le modèle médical fournit une base conceptuelle pour la pratique du service social.

\section{La société moderne et les pratiques " paternalistes " auprès des familles}

Avec l'avènement de la société post-industrielle, de nouvelles pratiques parentales voient le jour sous l'influence de la reconnaissance de la fragilité de l'enfance et de la responsabilité éducative des parents (Laurendeau, 1985). On assiste à une préoccupation grandissante pour le bien-être de l'enfant et la responsabilité des parents, et particulièrement des mères, s'en trouve considérablement augmentée. L'organisation familiale passe alors d'un modèle patricentré à un modèle centré sur l'enfant (Neyrand, 2003). L’influence de la nouvelle puériculture «à la docteur Spock » et la vulgarisation des ouvrages de Freud et de Piaget entraînent une préoccupation grandissante chez les parents pour le développement affectif et intellectuel de leurs enfants (Laurendeau, 1985). La petite enfance est de plus en plus reconnue comme étant le moment critique de la formation des futurs adultes et les valeurs associées à l'éducation des enfants changent considérablement. La stimulation, l'affection et le contact physique sont désormais les meilleurs atouts pour assurer le bonheur présent et futur du jeune enfant et les notions d'interdit, de discipline et d'autorité perdent de leur importance (Gauthier et Bujold, 1992). La reconnaissance des besoins de l'enfant et de l'influence différenciée des parents modifie le rôle du père à qui l'on reconnaît davantage d'influence sur le développement psychosocial de ses enfants. Cette nouvelle façon de concevoir le rôle du père n'est pas sans conséquence chez les hommes à qui l'on demande d'être plus présents auprès des enfants pour assumer un rôle de socialisation, alors que leur rôle de pourvoyeur les oblige à passer de nombreuses heures à l'extérieur du foyer (Deslauriers, 2002).

\section{Le rôle de l'État dans le contrôle des pratiques parentales}

Avec l'importance plus grande accordée à la période de l'enfance, les nombreuses exigences qui pèsent sur les parents et l'isolement de plus en plus grand des familles, l'État établit des normes en matière d'éducation et se substitue graduellement à la compétence 
exclusive de l’Église et de la famille en la matière (Laurendeau, 1985; Robinette, 1995; Roy, Lépine et Robert, 1990). La Grande Dépression des années 1930, la Seconde Guerre mondiale et les bouleversements qui en découlent entraînent un questionnement général des rôles respectifs de l'État et de l'initiative privée en matière de bien-être social et d'assistance aux personnes vulnérables. En 1937, le gouvernement unioniste fait adopter par l'Assemblée législative une loi qui vient en aide aux mères nécessiteuses, des mères dont le nombre augmente en flèche depuis le début de la crise économique. Cette loi marque un tournant dans l'histoire des politiques sociales au Québec et annonce une plus grande prise en charge par l'État de ce qui était autrefois assumé par les œuvres de charité (Vaillancourt, 1988). La loi sur la fréquentation scolaire illustre également le rôle grandissant de l'État dans l'éducation des enfants en imposant des amendes aux parents qui retirent leurs enfants de l'école avant l'âge permis. À partir des années 1960, d'autres politiques sociales sont mises de l'avant et intensifient la présence de l'État dans la vie de moins en moins privée des familles. Il existait déjà depuis le début des années 1950, les agences diocésaines de service social qui, en plus d'assurer la distribution de certaines allocations sociales, avaient comme préoccupation de protéger les enfants et d'aider les familles (Joyal, 1999), mais la deuxième moitié du siècle va marquer un tournant. Un nouvel ensemble législatif se développe par l'adoption d'une loi-cadre régissant l'organisation des services sociaux (1971) et par l'introduction de la Charte québécoise des droits (1975). Ces transformations entraînent des modifications législatives dans le domaine de l'enfance et de la famille qui débouchent sur une loi sur la protection de la jeunesse en 1977 (D’Amours, 1986). Les deux parents sont dorénavant placés sur un pied d'égalité quant à l'exercice de l'autorité sur leurs enfants mineurs, car la notion « d'autorité paternelle » est remplacée par celle "d'autorité parentale ». Dans une perspective de justice sociale, c'est désormais la protection de l'enfance, tant sur le plan physique que psychologique, qui justifie l'incursion de l'État dans la vie privée des familles.

\section{Des pratiques sociales axées sur le placement de l'enfant et l'éducation des parents}

La transformation des mesures juridiques et des politiques sociales qui a marqué cette période a fait en sorte que de nouveaux acteurs s’introduisent dans la vie des familles. En effet, médecins, psychologues, travailleurs sociaux et instituteurs jouent un rôle actif dans l'éducation des enfants (Laurendeau, 1985). À la faveur de l'extension de l'État-providence au Québec, les services publics se professionnalisent et prennent en charge les besoins de la population en disqualifiant souvent, du même coup, la contribution et les compétences des familles et des communautés (Roy, Lépine et Robert, 1990). Bien que la pratique du service social au Québec priorise officiellement l'intervention auprès des familles en qualifiant les premières agences de service social d' "agences de services à la famille ", les travailleurs sociaux de l'époque ne rencontrent pas les familles et limitent plutôt leurs interventions aux mères, ces dernières étant jugées plus « connaissantes » ou " importantes » pour assurer le bien-être de leurs enfants (Côté, 1991). Deux principales tendances marquent alors l'intervention auprès des parents jugés inaptes ou incompétents dans l'accomplissement de leur rôle auprès de leurs enfants : le recours au placement et l'éducation parentale dans une visée normative. 
Selon Durning (2002), le recours au placement est alors justifié par la nécessité de séparer l'enfant d'un milieu familial considéré comme incompétent, voire dangereux. Malgré la volonté clairement exprimée dans la loi sur la protection de la jeunesse de maintenir le plus possible l'enfant dans sa famille, le placement en famille d'accueil ou en centre d'accueil est plus fréquemment utilisé pour venir en aide aux enfants. Les travailleurs sociaux ont tendance à retirer les enfants de leur milieu familial et à les placer en centre ou en famille d'accueil sans que des motifs suffisants le justifient et sans que des mesures efficaces de soutien à la famille soient mises en place : "la proportion des enfants placés suit une courbe ascendante qui culmine en 1972 et fléchit par la suite jusqu'en 1993 » (Joyal et Chatillon, 1996 : 33). Mais le recours massif au placement est progressivement remis en question en raison de son coût, mais aussi des difficultés de réinsertion sociale des jeunes retirés de leur famille (Durning, 2002). Cette «tradition » du placement fut l'objet de multiples travaux de recherche en Europe, aux ÉtatsUnis et au Québec, travaux qui se sont penchés sur l'efficacité de cette mesure et ses effets non désirés sur les jeunes placés, notamment en mettant à jour les situations de maltraitance en institution (Fanshel et Shinn, 1978; Golstein, Freud et Solnit, 1979; Vachon, 1982). Ces études ont conduit à ne plus considérer systématiquement le placement comme une mesure de protection pour l'enfant. Selon Joyal et Chatillon (1996), trois grands facteurs d'ordre social et culturel ont influencé l'évolution particulière du Québec en ce qui concerne le recours au placement comme mesure d'aide aux enfants : (a) la lente évolution législative en matière de protection de l'enfance et de rapports familiaux (b) la place longtemps prépondérante de l'Église catholique dans le champ de l'assistance et (c) le développement relativement récent des savoirs et des pratiques dans le domaine de l'aide à l'enfance et à la famille.

Parallèlement à l'évolution du recours au placement, cette période est également marquée par le développement de l'éducation parentale dans une visée normative. Selon Clarke-Stewart (1998), les années 1960 témoignent d’une entrée en force des professionnels dans le domaine de l'éducation des enfants. Aux États-Unis, la mise en place des programmes préscolaires compensatoires Head Start et FollowThrough au milieu des années 1970 donne l'occasion aux chercheurs en éducation, en travail social, en psychologie et en sociologie, d'observer les processus familiaux in situ (Durning, 2002). L'ouvrage marquant de Brofenbrenner (1977), qui met l'accent sur les contextes multiples pour le développement de l'enfant (Allen, Sethi, Smith et Astuto, 2007), fait prendre conscience de l'importance d'inclure les parents dans l'intervention auprès de l'enfant. Les interventions axées sur l'éducation parentale se développent et offrent une alternative au placement (Durning, 2002). Au Québec, les écoles de parents mettent l'accent sur l'éducation familiale en se basant sur les nouveaux savoirs issus de la puériculture, de la psychologie et de la pédagogie (Lemieux et Comeau, 2002). Les parents des milieux défavorisés sont la cible d'intervention prioritaire, leur formation étant perçue comme la clé du développement optimal de leurs enfants. L’évaluation des compétences des parents et des ressources de leur milieu demeure cependant centrée sur les normes de la classe moyenne. Dans un tel contexte, le travailleur social adopte un rôle d'expert en identifiant les problèmes des clients ainsi que les changements nécessaires afin qu'ils deviennent des parents « socialement 
acceptables ». L’éducation parentale se présente donc à la fois comme un soutien à la parentalité et une forme de contrôle social.

En somme, au cours de cette période on remarque que la responsabilité des parents à l'égard des enfants augmente, alors que leur marge d'autonomie quant à la façon de les élever tend à se rétrécir; ils doivent moduler leurs relations avec leurs enfants selon les prescriptions des spécialistes qui sont de plus en plus nombreux à intervenir dans l'entretien, le soin et l'éducation des enfants (Robinette, 1995; Dandurand, 2001). À l’ère de l’État-providence, les spécialistes de l'enfant et de la famille, tels que les travailleurs sociaux, se substituent souvent aux parents en prenant en charge leurs difficultés (Pourtois, 1991). Ils abandonnent la référence aux traditions pour se réclamer de plus en plus des connaissances scientifiques en se positionnant dans un rôle d’experts. La rationalité, l’innovation et l'efficacité deviennent les références dominantes dans l’organisation des services.

\section{La société actuelle et les pratiques de partenariat}

Les transformations majeures que connaît actuellement la famille québécoise conditionnent la manière de vivre des parents ainsi que la façon dont ils s'acquittent de leurs responsabilités envers leurs enfants. Dans la société actuelle, la famille est dite plurielle étant donné la multiplication des modèles familiaux et la diversité des formes de vie familiale. Par conséquent, le rôle parental s'exerce dans une pluralité de structures et est influencé par de multiples changements sociaux : baisse de la fécondité qui entraîne une réduction de la taille des familles, diminution du nombre de mariages au profit de l'union libre, augmentation des divorces et des séparations qui entraîne une hausse du nombre de familles monoparentales et recomposées. Le fonctionnement interne de la famille est aussi bousculé par l'augmentation de la présence des femmes sur le marché du travail en raison de la nécessité d’un double revenu afin de pourvoir aux besoins de la famille (gouvernement du Québec, 1999) et la reconnaissance de plus en plus grande du rôle du père auprès de son enfant (Deslauriers, 2002). La signification sociale de l'enfant change, passant de l'enfant « fruit du destin » ou " prolongement de la vie » à " l'enfant-projet », choisi et planifié (Gauthier et Bujold, 1992). Selon Mackie (1990), l'enfant est valorisé à un point tel que les parents doutent de leur compétence à en avoir.

\section{La crise de I'État-providence et la responsabilisation des parents}

La crise de l’État-providence vient remettre en question la légitimité des interventions gouvernementales dans une foule de domaines. À partir du milieu des années 1970, l'économie se détériore et l'endettement de l'État s'accentue sans qu'il puisse lutter efficacement contre l'inflation et le chômage (Fortin, Giroux et Parent, 1990). Comme dans la plupart des pays occidentaux, l'État québécois restreint son rôle protecteur alors que, sous l'effet de la précarisation du travail et des transformations sociales, les besoins de soutien des familles sont de plus en plus grands (Dandurand, 2001). Au cours des années 1980, les mesures législatives touchent des catégories de personnes particulièrement vulnérables que l'on pense aux enfants, 
aux femmes violentées et aux familles monoparentales. Selon Mayer (2002), cette réorientation du rôle de l'État vise à transférer la prise en charge d'une grande partie des problèmes sociaux aux individus, aux familles, à la communauté et à l'entreprise privée. La désinstitutionnalisation et le "virage ambulatoire " sont " justifiés par un discours soulignant les avantages du cadre familial pour assumer le bien-être, sinon la guérison, de ces personnes et proclamant la nécessité pour les aidants naturels de se responsabiliser quant aux membres plus fragiles de la famille » (Baillargeon, 1996, p. 29). Devant la multiplication des problèmes familiaux, qui sont discutés sur la place publique et moins tolérés socialement, certaines mesures sont mises en place pour l'enfance et la famille. C'est ainsi que le législateur fédéral procède à une réforme en profondeur du régime applicable aux contrevenants mineurs et que le gouvernement québécois accentue sa présence dans le domaine des services de garde et des ressources aux enfants atteints de déficits ou éprouvant des difficultés d'adaptation ou d'apprentissage.

Sur le plan des politiques sociales, le gouvernement du Québec adopte «l'Énoncé de politique familiale » dans lequel il affirme son intention de mettre de l'avant une stratégie globale d'intervention en faveur de la famille et il crée le Conseil de la famille. Le projet de révision du Code civil met de l'avant l'égalité juridique des époux, l'abolition de toute discrimination entre enfants légitimes et naturels et la sauvegarde de l'intérêt de l'enfant dans toutes les décisions qui le concerne. L’obligation de contribuer aux charges du ménage est aussi étendue à l'union de fait afin de tenir compte des réalités nouvelles qui affectent le couple. Enfin, l'autorité parentale remplace la notion de puissance paternelle. Dans ce projet de révision du Code civil, Fortin, Giroux et Parent (1990) décèlent un plus grand contrôle de l'État sur les parents et constatent le passage du droit des parents sur l'enfant aux devoirs des parents envers celui-ci. Bref, la crise de l'État-providence conduit à l'adoption de politiques sociales qui visent à responsabiliser les parents dans leur rôle auprès de leurs enfants et à les mettre à contribution dans l'intervention (Robert, 1990). Dès lors, le parent devient un acteur incontournable pour le bien-être de son enfant et, en ce sens, l'État et les différents organismes qui en relèvent créent des programmes qui s'adressent prioritairement aux pères et aux mères.

\section{Des pratiques d'intervention axées sur le partenariat avec les familles}

Avec la récession économique qui touche tout le pays, le soutien financier accordé aux programmes sociaux diminue. Dès lors, parents et intervenants familiaux doivent réajuster leurs attentes et trouver de nouveaux moyens pour répondre aux besoins des jeunes et des familles (Clarke-Stewart, 1998). Le discours des gestionnaires et des intervenants change, misant davantage sur le développement d'une relation harmonieuse avec les parents et les familles (Bouchard, Pelchat et Boudreault, 1996). En outre, l'intervention sociale se transforme en misant sur une approche intégrée et renouvelée (Mayer, 2002). Selon Chamberland et al. (1996), la crise économique, l'aggravation des problèmes sociaux et le déploiement du modèle écologique incitent les chercheurs à critiquer l'efficacité des pratiques traditionnellement centrées sur les individus et les déficits personnels. De nouveaux modèles d'intervention se développent dans l'intervention auprès des parents. C'est le cas, notamment, des approches axées sur 
l'empowerment, de l'intervention basée sur les forces et les compétences du système familial, de la perspective écologique ainsi que de l'action basée sur l'utilisation du soutien social (Mayer, 2002). Bref, la participation des parents à l'intervention est officiellement recherchée et la reconnaissance de leurs forces et compétences est de plus en plus inscrite dans les pratiques ${ }^{5}$.

Dans le domaine de la famille et de l'enfance, des interventions préventives et curatives se développent afin de trouver des solutions de rechange au placement. Des interventions de réadaptation se structurent et se diversifient pour répondre plus adéquatement aux besoins des jeunes et des familles. Le discours théorique qui sous-tend l'intervention auprès des parents se transforme, même si le changement d'orientation semble plus influencé par des raisons idéologiques et financières que par les connaissances scientifiques (Wald, Carlsmith, Leiderman, Smith et De Sales French, 1988). Dorénavant, les problèmes des jeunes et des familles ne peuvent plus être appréhendés en tenant compte uniquement de leurs manifestations individuelles et familiales; le système familial doit être analysé dans son environnement. Garant (1992) note que de nombreux chercheurs sont d'avis que des mesures préventives et curatives qui ne s’appuieraient pas sur un développement économique et social sont vouées à l'échec.

Le thème de la prise en charge des problèmes sociaux par le milieu devient omniprésent et domine bon nombre d'interventions (Mayer, 2002). Alors que les interventions des travailleurs sociaux ont pendant longtemps contribué à séparer l'enfant de sa famille afin que son éducation se poursuive dans un milieu protégé, les actions récentes cherchent plutôt à prévenir le placement en intervenant directement dans le milieu familial à l'aide d'interventions préventives et curatives. Plusieurs programmes d'intervention précoce visant l'ensemble de la famille voient le jour. En effet, chercheurs et intervenants ont réalisé qu'il est relativement inefficace de se concentrer uniquement sur l'enfant; des actions efficaces exigent que les membres de sa famille, en particulier les parents, changent leurs façons de faire. Sur le plan curatif, de nombreux programmes de soutien intensif aux familles sont mis sur pied (Carrier, Saint-Jacques, Chabot et Thibault, 1992; Dagenais et Bouchard, 1996; Garant, 1992). Ces programmes prennent place lorsqu'un problème grave et susceptible de mener à un placement de l'enfant est décelé dans la famille. Malgré la diversification des méthodes d'intervention, ils conservent toute leur pertinence, car le placement en milieu substitut continue d’être fréquemment utilisé pour venir en aide aux enfants se trouvant sous la protection de l’État (Conseil des affaires sociales, 1990).

5. Bien que certains auteurs soulignent que l'utilisation des forces du client est un principe d'action qui se retrouve dans les écrits classiques du travail social (Bendor, Davidson et Skolnik, 1997; Cohen, 2002), plusieurs situent son origine plus récemment, vers le début des années 1980 (Rapp et Chamberlain, 1985; Staudt, Howard et Drake, 2001; Weick, 1983; Werrbach, 1996). 


\section{CONCLUSION}

Cet article a tenté de démontrer que les changements dans les pratiques d'intervention auprès des parents sont attribuables, dans une large mesure, à des changements sociétaux. Si historiquement ils ont été longtemps laissés à eux-mêmes avant d’être ensuite jugés incompétents, les parents sont actuellement réinvités à exercer leurs compétences parentales (Robert, 1990) à l'intérieur de paramètres fixés par les normes sociales. On se retrouve donc dans une réalité où s'opposent une vision privée de la famille et un contrôle social de l'État sur cette institution. Si la responsabilité de l'enfant est confiée aux parents, l'État se réserve le droit de s’introduire dans les familles pour sanctionner des pratiques éducatives jugées inacceptables. Dans ce rapport entre la famille et l'État, les intervenants sociaux jouent le rôle de médiateurs en privilégiant des pratiques d'intervention qui tentent de remettre les parents au centre du processus tout en leur apportant un soutien dans l'exercice de leur rôle parental. Dans un tel contexte, la finalité de l'intervention n'est pas d'apprendre aux parents ce qu'ils doivent faire afin de mieux élever leurs enfants, mais plutôt de viser leur empowerment et le développement de leurs forces et de leurs compétences. Toutefois, bien que certains changements d'attitudes et de langage soient apparus au cours des dernières années, Noble, Perkins et Fatout (2000) affirment que la focalisation sur les problèmes et les pathologies demeure particulièrement présente dans les services sociaux, notamment dans les services destinés aux enfants. Terrisse et Larose (2002) soulignent que les changements que l'on remarque dans le discours des intervenants sont loin d'être toujours observés dans les faits, car les nouvelles philosophies d'intervention auprès des familles en difficulté se heurtent à des attitudes profondément enracinées chez les professionnels et les parents. Les premiers ont souvent la conviction d'être les détenteurs quasi exclusifs du savoir, alors que les parents sont habitués à maintenir à leur égard une relation de dépendance et non à établir un partenariat sur une base égalitaire. Ce constat entraîne certains questionnements sur la pratique du service social. Ainsi, on peut se demander si les intervenants qui agissent dans le prolongement de l'État disposent d'une marge de manœuvre pour dénoncer les conditions matérielles dans lesquelles se retrouvent certaines familles. Est-ce qu'un discours qui reconnaît les compétences des familles et privilégie la collaboration est compatible avec des pratiques axées sur les déficits et inscrites dans une dynamique d'opposition? Est-ce que les efforts conceptuels pour élargir les modèles d'action auprès des familles en difficulté se répercutent vraiment sur les pratiques mises de l'avant?

L’évolution du travail social a de tout temps été marquée par une tension entre l'action sociale, axée sur la diminution des iniquités, et l'aide individuelle basée sur les ressources et les compétences des personnes. La dynamique générée par cette tension devrait permettre au travail social de continuer d'ajuster ses pratiques pour répondre simultanément aux exigences de la responsabilité collective et des droits individuels des jeunes, des parents et des familles. 


\section{BiBLIOGRAPHIE}

Allen, L., A. Sethi, S. Smith, et J. Astuto (2007). « Parent education: Lessons inspired by Heart Start ", Child development and social policy: knowledge for action, American psychological association, Washington, p. 219-231.

ARIÈs, P. (1973). L'enfant et la vie familiale sous l'ancien régime, $2^{\mathrm{e}}$ édition. Paris, Seuil.

BAillargeon, D. (1996). "Les politiques familiales au Québec. Une perspective historique », Lien social et politique - RIAC, $\mathrm{n}^{0}$ 36, p. 21-32.

Bendor, S., K. DAVIDSON, et L. SKOLNIK (1997). « Strengths-pathology dissonance in the social work curriculum », Journal of teaching in social work, vol. 15, n⿳0 1-2, p. 3-16.

BERG, I.K. (1996). Services axés sur la famille: Une approche centrée sur la solution, Ramonville, Érès.

Berg, I.K., et S. Kelly (2001). Des solutions à inventer dans les services à l'enfance, New York, Edisem.

Bouchard, J.-M., D. Pelchat, et P. Boudreault (1996). " Les relations parents et intervenants : Perspectives théoriques », Apprentissage et Socialisation, vol. 17, $\mathrm{n}^{0} 1-2$, p. 21-34.

CARISSE, C. (1974). La famille: Mythe et réalité québécoise, Québec, Conseil des affaires sociales et de la famille.

Carrier, G., M.-C. Saint-Jacques, L. Chabot, et M. Thibeault (1992). " Les services intensifs : une nouvelle approche dans l'intervention auprès des familles à risque ", Revue Service social, vol. 41, p. 41-75.

Chamberland, C., N. Dallaire, L. Fréchette, J. Lindsay, J. Hébert, et S. Cameron (1996). " Les dimensions sociales de la prévention et de la promotion du bien-être des jeunes et de leur famille: un portrait des pratiques au Québec », Nouvelles pratiques sociales, vol. $9, \mathrm{n}^{\mathrm{0}} \mathrm{2}$, p. 65-86.

Снавот, М. (1989). «Les discours sur la famille », Rôles et valeurs familiales, Document $\mathrm{n}^{0}$ 3, Québec, Musée de la civilisation.

ClARKE-STEWART, K.A. (1998). « Historical shifts and underlying themes about rearing young children in the United States: Where have we been? Where are we going? », Early Development and Parenting, vol. 7, n 2, p. 101-117.

CôTÉ, C. (1991). « La thérapie familiale : Évolution et enjeux », Intervention, nº 89, p. 5-8.

Dagenais, C., et C. Bouchard (1996). «Recension des écrits concernant l'impact des programmes de soutien intensif visant à maintenir les enfants et adolescents dans leur famille », Revue canadienne de santé mentale communautaire, vol. 15, nº 1, p. 63-82. 
D’Amours, O. (1986). « Survol historique de la protection de l'enfance au Québec, de 1608 à 1977 », Revue Service social, vol. 3, p. 386-415.

DANDURAND, R. B. (2001). « Familles et services sociaux : Quelles limites aux interventions? », Revue Service social, vol. 48, p. 1-15.

Deslauriers, J.-M. (2002). « L’évolution du rôle du père au Québec », Intervention, n 116, p. 52-61.

DuRning, P. (2002). « De la substitution à la formation parentale. Émergence d’une approche socio-éducative de la parentalité », dans D. FABLET (dir.), Les interventions socioéducatives : Actualité de la recherche, Collection Savoir et Formation, Paris, L’Harmattan.

FAnshel, D., et E. Shinn. (1978). Children in foster care, New York, Columbia University Press.

ForTin, A. (1987). Histoire de familles et de réseaux: La sociabilité au Québec d'hier à demain, Montréal, Éditions Saint-Martin.

Fortin, P., G. Giroux, et P.-P. PARent (1990). Le pouvoir et la légitimité de l'intervention de l'État auprès des familles en lien avec l'autorité et la compétence parentale dans le contexte des législations et des pratiques existantes: Approche éthique, Québec, Conseil de la famille, Groupe de recherche Éthos.

GARANT, L. (1992). Les programmes de soutien familial : Une alternative au placement des jeunes?, Québec, Ministère de la Santé et des Services sociaux, Direction générale de la planification et de l'évaluation.

GAUTHIER, M., et J. Bujold (1992). « L’enfance au Québec : Une analyse des tendances », dans G. Pronovost (dir.), Comprendre la famille, Actes du 1er Symposium québécois de recherche sur la famille, Sainte-Foy, Presses de l’Université du Québec, p. 391-407.

Golstein, J., A. Freud, et A. Solnit (1979). Before the best interest of child, New York, Free Press.

GOUVERNEMENT DU QUÉBEC (1999). Un portrait statistique des familles et des enfants au Québec, Québec, Ministère de la Santé et des Services sociaux.

Joyal, R. (2000). L'évolution de la protection de l'enfance au Québec, Sainte-Foy, Presses de l’Université du Québec.

Joyal, R. (1999). Les enfants, la société et l'État au Québec, Éditions Hurtubise HMN, Cahiers du Québec.

Joyal, R., et C. Chatillon (1996). « Le placement des enfants au Québec, des années trente à aujourd'hui. Une mesure trop souvent utilisée? Constatations et hypotheses », Revue Service social, vol. 45, $\mathrm{n}^{0}$ 2, p. 31-50. 
Laurendeau, F. (1985). «Types de sociétés et de prises en charge : La professionnalisation de l'élevage des enfants », dans J. Dufresne, F. Dumont et Y. MARTin (dir.), Traité d'anthropologie médicale, Montréal, PUQ /IQRC/PUL.

Lemieux, D., et M. Comeau (2002). Le mouvement familial au Québec, 1960-1990 : Une politique et des services pour les familles, Sainte-Foy, Presses de l’Université du Québec.

MACKIE, C. (1990). «Socialization: Changing views of child rearing and adolescence », dans Families, Toronto, McGraw-Hill, p. 115-148.

MAYER, R. (2002). Évolution des pratiques en service social, Boucherville, Gaëtan Morin Éditeur.

NEYRAND, G. (2003). «L'évolution du regard sur la relation parentale: L'exemple de la France », Nouvelles pratiques sociales, vol. 16, $\mathrm{n}^{\mathrm{o}} 1$, p. 27-44.

Noble, D.N., K. Perkins, et M. FATOUT (2000). « On being a strength coach: Child welfare and the strengths model », Child and Adolescent Social Work Journal, vol. 17, n 2, p. 141153.

Pourtois, J.-P. (1991). Innovation en éducation familiale, Montréal, Éditions du renouveau pédagogique.

Provencher, J. (1988). Les quatre saisons dans la vallée du Saint-Laurent, Montréal, Boréal.

RoBert, L. (1990). «Le rapport entre l'État et la famille : Caractéristiques et enjeux », dans ROY, J., R. LÉPINE et L. ROBERT, État et famille : des politiques sociales en mutation, Sainte-Foy, Université Laval, CRSC.

RobinetTe, C. (1995). Compétence parentale: Son évolution et ses définitions actuelles, mémoire de maîtrise, Université de Sherbrooke.

ROY, J., R. LÉPINE, et L. ROBERT (1990). État et famille : des politiques sociales en mutation, Sainte-Foy, Université Laval, CRSC.

RYCUS, J.S., et R.C. Hughes (1998). Field guide to child welfare, Washington, CWLA Press.

TERrisse, B., et F. LAROSE (2002). «L’intervention socio-éducative précoce auprès de la famille », dans les actes du colloque Difficulté d'apprendre, difficulté d'enseigner, Luxembourg, Ministère de l'Éducation nationale, de la Formation professionnelle et des Sports.

VAILlancourt, Y. (1988). L’évolution des politiques sociales au Québec, 1940-1960, Montréal, Presses de l’Université de Montréal.

VACHON, J. (1982). Enfants en soins d'accueil : cinq ans après leur placement, Université Laval, École de service social, Laboratoire de recherche.

Wald, M.S., J.M. Carlsmith, P.H. Leiderman, C. Smith, et R. De Sales French (1988). Protecting abused and neglect children, Stanford, Stanford University Press. 